\title{
A Recursive Way for Sparse Reconstruction of Parametric Spaces
}

\author{
Oguzhan Teke ${ }^{1}$, Ali Cafer Gurbuz ${ }^{2}$, Orhan Arikan ${ }^{1}$ \\ Department of Electrical and Electronics Engineering \\ Bilkent University ${ }^{1}$, TOBB University of Economics and Technology ${ }^{2}$ \\ E-mail: teke@ee.bilkent.edu.tr, acgurbuz@etu.edu.tr, oarikan@ee.bilkent.edu.tr
}

\begin{abstract}
A novel recursive framework for sparse reconstruction of continuous parameter spaces is proposed by adaptive partitioning and discretization of the parameter space together with expectation maximization type iterations. Any sparse solver or reconstruction technique can be used within the proposed recursive framework. Experimental results show that proposed technique improves the parameter estimation performance of classical sparse solvers while achieving Cramér-Rao lower bound on the tested frequency estimation problem.

Index Terms-Compressive sensing, basis mismatch, off-grid
\end{abstract} targets, recursive solver, sparse reconstruction.

\section{INTRODUCTION}

$\mathbf{S}^{\mathrm{p}}$ PARSE signal representations and the theory of compressive sensing (CS) [1], [2] has received considerable attention in many research communities and has a wide range of applications. CS states that a sparse signal in some known basis can be efficiently acquired using a small set of nonadaptive and linear measurements. The classical CS approaches assume a pre-defined known sparsity basis and mainly focuses on solution of an underdetermined linear systems such as:

$$
\boldsymbol{y}=\boldsymbol{\Phi} \boldsymbol{\Psi} \boldsymbol{x}+\boldsymbol{n}
$$

where $\boldsymbol{y} \in \Re^{M}$ and $\boldsymbol{n} \in \Re^{M}$ are the measurement and noise vectors of dimension $\mathbf{M} . \boldsymbol{\Psi}$ is the a $N \times N$ basis matrix and $\boldsymbol{\Phi} \in \Re^{M \times N}$ is the compressive measurement matrix where $M<N$. Since the sparsity basis $\boldsymbol{\Psi}$ is assumed to be known, length of the sparse signal $N$ is also fixed. Hence in classical CS, the number of measurements is considered as a free parameter and analysis on the required number of measurements for given sparsity levels are done. Proven bounds are also obtained with conditions on $A=\Phi \Psi$.

In practical systems, even though it is known that the given signal has a sparse representation in some continuous parametric space, an exact predefined basis may not be known, even it may not exist. For example in the frequency estimation problem, assume a given set of observations consist of a few number of frequency components. However, those frequencies can be anything from the continuous range. In this case, it is not possible to use the classical model of $\boldsymbol{y}=\boldsymbol{A} \boldsymbol{x}$ for an arbitrary observation vector with fixed basis. Even though $\boldsymbol{y}$ has a sparse representation, actual parameters lie in a continuous domain and cannot be exactly represented by a finite length sparse vector $\boldsymbol{x}$. In order to utilize sparse

This work was supported by TUBITAK grant with project number $113 \mathrm{E} 515$. solvers, mere attempt to use a matrix-vector model requires the discretization of the parameter space. If discretization creates $N$ distinct points, then sparse parameters are approximated by the vector $\boldsymbol{x}$. In general approximation does not hold very well and off-grid problem is introduced. In the literature, the effect of this basis mismatch has been observed and analyzed in several studies [3]-[6].

Unlike the classical approach, in a practical problem analysis starts with a given set of observations $\boldsymbol{y}$, hence $M$ is fixed. Since discretization resolution determines the length of the sparse signal, $N$ becomes a design parameter. The discretization level, hence $N$, should be determined together with number of measurement $M$ and sparsity level $K$ in order to achieve required incoherency in the obtained dictionary $\boldsymbol{A}$. Dense discretization of the parameter space violates the restricted isometry property (RIP) [7]. As a result, a fixed discretization of the parameter space is not suitable for an arbitrary observation. Dictionary should be constructed according to the set of observations and the sparsity level of the signal.

\section{Compressive Sensing And Continuous Signal SPACES}

In a practical system, observation can be modeled as follows:

$$
y(t)=\sum_{i=1}^{K} \alpha_{i} \psi\left(\theta_{H_{i}} ; t\right)+n(t),
$$

where $\psi\left(\theta_{H_{i}} ; t\right)$ is a component of the observation $y(t)$ corresponding to parameter $\theta_{H_{i}}, \alpha_{i}$ is the scale for the $i^{t h}$ component and $n(t)$ is the measurement noise. In this model, signal atom $\psi(\theta ; t)$ is a parametric function that may depend on one or more parameters. In a delay-Doppler radar application, for example, $\psi\left(\theta_{H_{i}} ; t\right)$ is defined as follows:

$$
\psi(\theta ; t)=s(t-\tau) e^{-j 2 \pi \nu t},
$$

where $s(t)$ is the transmitted signal and $\theta=[\tau, \nu]$ is 2 dimensional continuous parameter space in which $\tau$ and $\nu$ corresponds to delay and Doppler shift, respectively. In the frequency estimation problem signal atoms are complex exponentials, hence $\psi\left(\theta_{H_{i}} ; t\right)$ can be defined as follows:

$$
\psi(\theta ; t)=e^{-j 2 \pi f t},
$$

where one dimensional $\theta=f$ corresponds to frequency. 
To use efficient digital signal processing techniques, samples of the continuous model in (2) are obtained. For $t \in \Re^{M}$, vector holding the sampling times in $[0, T]$ interval, sampled data model can be written as follows:

$$
\boldsymbol{y}=\sum_{i=1}^{K} \alpha_{i} \psi\left(\theta_{H_{i}} ; \boldsymbol{t}\right)+\boldsymbol{n} .
$$

This form can be reduced to commonly used compressive sensing setup by discretizing the continuous and bounded parameter space, $\mathcal{P}$. For a more abstract formulation that will be useful in the presentation of the proposed approach, let $d(\cdot, \cdot)$ be a functional that takes the continuous space $\mathcal{P}$ and a discretization interval $\lambda_{\theta}$ and returns a set of discrete parameter points:

$$
\left\{\theta_{1}, \theta_{2}, \ldots, \theta_{N}\right\}=d\left(\mathcal{P}, \lambda_{\theta}\right) .
$$

This discretization provides $N$ grid points, $\theta_{i} \in \mathcal{P}$, for $1 \leqslant$ $i \leqslant N$. For each $\theta_{i}$, the $M$-dimensional corresponding signal atom is computed using the given sampling times as:

$$
\boldsymbol{a}_{i}=\psi\left(\theta_{i} ; \boldsymbol{t}\right)
$$

By using (7) for each $\theta_{i}, M \times N$ dimensional dictionary is constructed as $\boldsymbol{A}=\left[\boldsymbol{a}_{1}, \boldsymbol{a}_{2}, \ldots, \boldsymbol{a}_{N}\right]$. Note that the dictionary composed with the discretization of the parameter space has to guarantee the reconstruction of arbitrary $K$-sparse signal in the column space of $\boldsymbol{A}$. Each CS technique has its own guarantees for the recovery. For simplicity in the development, we will assume that OMP is used as the sparse solver. For an alternative CS reconstruction technique, it is straightforward to make proper changes in the following development.

Recovery guarantees of OMP has been discussed in the literature. In [8], a sufficient condition for the recovery of a $K$-sparse signal in terms of mutual coherence is provided as:

$$
\mu(\boldsymbol{A}) \leqslant \frac{1}{2 K-1},
$$

where $\mu(\boldsymbol{A})$ is:

$$
\mu(\boldsymbol{A})=\max _{k \neq l} \frac{\left|\boldsymbol{a}_{k}^{H} \boldsymbol{a}_{l}\right|}{\left\|\boldsymbol{a}_{k}\right\|_{2}\left\|\boldsymbol{a}_{l}\right\|_{2}} .
$$

Notice that mutual coherence is a functional of the basis vectors defined in (7), hence it has to be computed accordingly. To illustrate the effect of sufficient recovery condition on the discretization of the continuous parameter space, we will concentrate on the frequency estimation problem, hence complex exponentials defined in (4) will be used as the basis functions.

Let the discretization interval between two adjacent discrete parameter points be $\lambda_{f}$, hence $f_{l+k}-f_{l}=k \lambda_{f}$. In this case, normalized inner product of basis vectors corresponding two grid points can be computed as:

$$
\begin{aligned}
\frac{\left|\boldsymbol{a}_{k}^{H} \boldsymbol{a}_{l}\right|}{\left\|\boldsymbol{a}_{k}\right\|_{2}\left\|\boldsymbol{a}_{l}\right\|_{2}} & =\frac{\left|\boldsymbol{a}\left(f_{k} ; \boldsymbol{t}\right)^{H} \boldsymbol{a}\left(f_{l} ; \boldsymbol{t}\right)\right|}{\left\|\boldsymbol{a}\left(f_{k} ; \boldsymbol{t}\right)\right\|_{2}\left\|\boldsymbol{a}\left(f_{l} ; \boldsymbol{t}\right)\right\|_{2}}=\frac{1}{M}\left|\sum_{i=1}^{M} e^{j 2 \pi(k-l) \lambda_{f} t_{i}}\right| \\
& \approx\left|\operatorname{sinc}\left((k-l) \lambda_{f} T\right)\right|,
\end{aligned}
$$

which is a close approximation when the sampling instants have uniform distribution in $[0, T]$. Since $|\operatorname{sinc}(x)| \geqslant$ $|\operatorname{sinc}(n x)|$ holds true for all non-zero real $x$ and non-zero integer $n$, mutual coherence of the dictionary is found as:

$$
\mu(\boldsymbol{A})=\max _{k \neq l}\left|\operatorname{sinc}\left((k-l) \lambda_{f} T\right)\right|=\left|\operatorname{sinc}\left(\lambda_{f} T\right)\right| .
$$

In order to guarantee that OMP will recover a $K$-sparse solution, the discretization interval $\lambda_{f}$ has to satisfy the condition given in (8) resulting in the following inequality:

$$
\mu(\boldsymbol{A})=\left|\operatorname{sinc}\left(\lambda_{f} T\right)\right| \leqslant \frac{1}{2 K-1} .
$$

Assuming $0 \leqslant \lambda_{f} T \leqslant 1$, we can safely take the inverse of the sinc function. Hence, the smallest possible discretization interval for the frequency estimation problem that OMP is guaranteed to recover a $K$-sparse signal is:

$$
\lambda_{f}(K)=\frac{1}{T} \operatorname{sinc}^{-1}\left(\frac{1}{2 K-1}\right) .
$$

Since $\operatorname{sinc}^{-1}(x)$ is a monotonic decreasing function for $0 \leqslant$ $x \leqslant 1$, for larger $K$ values $\lambda_{f}(K)$ becomes larger as well. This implies that for less sparse signals, we have to use a coarser discretization in the continuous parameter space resulting in more severe performance degradation due to off-grid problem. Fig. 1 shows the discretization intervals as a function of $K$.

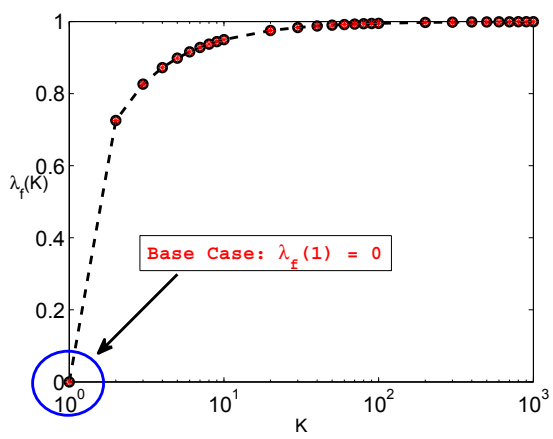

Fig. 1. Lower bound of feasible discretization interval with respect to sparsity level that guarantees the recovery of the sparse signal in the frequency estimation problem, using OMP as the sparse solver.

As expected, the discretization interval is an increasing function of $K$. Also, we observe that the discretization interval is always less than $1 / T$. It is known that if a system with classical sampling procedure takes samples in the $[0, T]$ time range, its frequency resolution is $1 / T$. Having a discretization interval smaller than $1 / T$ in the least sparse case is consistent with this fact. Finally, we observe that the allowed interval for 1-sparse case is 0 . In other words, for 1-sparse signals discretization interval can be set arbitrarily small, yet the recovery is still guaranteed in the noiseless setting. This last fact will provide the foundation of the proposed recursive approach presented in the following section.

It is important to note that (13) provides the allowed minimum interval for the frequency estimation problem when OMP is used as the sparse solver. The allowed interval for 
another solver or basis function may differ. In a more general and abstract sense, the required discretization interval can be represented as follows:

$$
\lambda_{\theta}=f(M, K) .
$$

Here, the function $f(\cdot, \cdot)$ can be computed analytically or numerically depending on the recovery condition imposed by the solver of choice on the basis functions. However, the observations based on the OMP case are valid for other solvers.

\section{PROPOSED RECURSIVE COMPRESSIVE SENSING FRAMEWORK}

For the model given in (2), for a given measurement vector $\boldsymbol{y} \in \mathcal{C}^{M}$ and a provisional estimate of the sparsity level $K$, a sparse solver can be written in the following abstract form:

$$
\left[\boldsymbol{\alpha}^{*}, \boldsymbol{\theta}^{*}\right]=\mathcal{S}(\boldsymbol{y}, K, \mathcal{P}),
$$

where $\boldsymbol{\theta}^{*}$ is the estimated parameter values, $\boldsymbol{\alpha}^{*}$ are the corresponding representation coefficients and $\mathcal{P}$ is the bounded and continuous parameter space. A dictionary is required in order to utilize CS based solvers in (15). At this step, the first thing is to determine the finest discretization interval dictated by (14). Then the parameter space $\mathcal{P}$ is discretized accordingly and the dictionary $\boldsymbol{A}$ is constructed using (7). In this way, we can define the problem as $\boldsymbol{y}=\boldsymbol{A} \boldsymbol{x}+\boldsymbol{n}$ and solve for the $K$ sparse reconstruction of the signal for a selected sparse solver of choice yielding $\boldsymbol{\alpha}^{*}$ and $\boldsymbol{\theta}^{*}$. Using $\left[\boldsymbol{\alpha}^{*}, \boldsymbol{\theta}^{*}\right]$, the observation vector can be represented as:

$$
\boldsymbol{y}=\sum_{i=1}^{K} \alpha_{i}^{*} \psi\left(\theta_{i}^{*} ; \boldsymbol{t}\right)+\boldsymbol{n},
$$

where $\boldsymbol{n}$ corresponds to the fit error of the sparse solver. The important thing to notice is that the matrix-vector model is an approximate relationship due to discretization of the parameter space. However, if the problem is highly sparse, this approximate relationship has a relatively high accuracy since the allowed discretization interval in (14) is a decreasing function of the sparsity. Therefore our main purpose is to split the $K$-sparse problem into set of smaller problems with higher sparsity. For this purpose, assume that we divide the problem into $c$ partitions as follows:

$$
\boldsymbol{y}=\sum_{i=1}^{K_{1}} \alpha_{1, i}^{*} \psi\left(\theta_{1, i}^{*} ; \boldsymbol{t}\right)+\ldots+\sum_{i=1}^{K_{c}} \alpha_{c, i}^{*} \psi\left(\theta_{c, i}^{*} ; \boldsymbol{t}\right)+\boldsymbol{n},
$$

where $\sum_{j=1}^{c} K_{j}=K, \bigcup_{j, i} \alpha_{j, i}^{*}=\boldsymbol{\alpha}^{*}$ and $\bigcup_{j, i} \theta_{j, i}^{*}=\boldsymbol{\theta}^{*}$. This process also partitions the given parameter space $\mathcal{P}$ into disjoint sets such that $\mathcal{P}=\bigcup_{j} \mathcal{P}_{j}$ and $\mathcal{P}_{j} \cap \mathcal{P}_{k}=\varnothing$ if $j \neq k$ with $\theta_{j, i}^{*} \in \mathcal{P}_{j}$. Expectation-Maximization (E-M) based frameworks provide an effective solution for the partitioned problems [9], [10]. Assuming the estimates of the last $c-1$ partitions' parameters are precise enough, we can construct the observation vector corresponding to those partitions and then find the partial observation vector corresponding to the first partition. This is the E-step of the framework. The M-step is to solve the problem with partial observation vector in its corresponding domain.

In general, in the E-step for an arbitrary $l^{\text {th }}$ partition, the corresponding partial observation vector is written as:

$$
\boldsymbol{y}_{l}=\boldsymbol{y}-\sum_{\substack{j=1 \\ j \neq l}}^{c} \sum_{i=1}^{K_{j}} \alpha_{j, i}^{*} \psi\left(\theta_{j, i}^{*} ; \boldsymbol{t}\right)
$$

In the M-step, in order to estimate parameters with higher accuracy, we solve the sparse reconstruction problem of observation vector $\boldsymbol{y}_{l}$ with sparsity estimation $K_{l}$ in the domain $\mathcal{P}_{l}$. Therefore, M-step of the framework is written as the solution of the following problem,

$$
\left[\boldsymbol{\alpha}_{l}^{*}, \boldsymbol{\theta}_{l}^{*}\right]=\mathcal{S}\left(\boldsymbol{y}_{l}, K_{l}, \mathcal{P}_{l}\right)
$$

Iteratively solving (18) and (19) from $l=1$ to $l=c$ realizes one pass of the EM approach. The most important thing to notice in the transformation of (15) to (19) under the EM framework is that the problems are identical to each other in the structural sense: all take an observation vector, provisional sparsity level and a parameter space to operate on. Thus, the approach used in the solution of (15) can also be applied to (19). Successively applying the very same approach to each sub-problem, the main problem splits itself into smaller, and sparser sub-problems with denser discretization of the parameter space in a recursive manner. The remarkable advantage of this approach lies in the reduction of the sparsity levels in the sub-problems. In the fragmentation from (15) to (19), the immediate observation is that $K_{l}<K$ for all $1 \leqslant l \leqslant c$ provided that $c>1$. Due to decreasing characteristics of (14), finer discretization of corresponding parameter spaces can be performed in the sub-problems, which improves the accuracy of the parameter estimations.

The proposed EM based recursive solution approach is summarized in Table I. Some of the steps are taken general so that the framework can be utilized in a wide range of problems with different characteristics specific to implementation of those steps. Note that the 5.2.2 step of the algorithm calls the same technique with partition $l$, hence the algorithm is recursive in this sense. In the following sub-sections, we will elaborate on the individual steps for clarity.

\section{A. Base Case of the Recursion}

Separation of the problem will be terminated in a finite amount of recursive calls since the sparsity of the main problem $K$ is finite. The most interesting case, which is also the base case of the recursion, happens when the sparsity of that partition reduces to one. Contrary to Fig. 1, discretization interval is lower-bounded by the Cramér-Rao lower bound (CRLB) in the noisy setting. This case will be illustrated on the frequency estimation problem using basis functions given in (4). In this model, observations are in the following structure in the 1-sparse case:

$$
\boldsymbol{y}=\alpha e^{j \phi} e^{j \omega \boldsymbol{t}}+\boldsymbol{n},
$$

where $\alpha, \phi$ and $\omega$ are the unknown amplitude, phase and 
TABLE I

Signature: $[\hat{\boldsymbol{\alpha}}, \hat{\boldsymbol{\theta}}]=\mathcal{S}(\boldsymbol{y}, K, \mathcal{P})$

1) $\lambda_{\theta}=f(M, K)$, find the required discretization interval,

2) $\left\{\theta_{1}, \ldots, \theta_{N}\right\}=d\left(\mathcal{P}, \lambda_{\theta}\right)$, discretization,

3) $\boldsymbol{A}=\left[\psi\left(\theta_{1} ; \boldsymbol{t}\right) \ldots \psi\left(\theta_{N} ; \boldsymbol{t}\right)\right]$, construct the dictionary,

4) $[\boldsymbol{\alpha}, \boldsymbol{\theta}]=$ SparseSolver $(\boldsymbol{y}, \boldsymbol{A}, K)$, a provisional solution,

5) While $[\boldsymbol{\alpha}, \boldsymbol{\theta}]$ is not a satisfactory solution,

5.1) $\left\{\left(\boldsymbol{\alpha}_{1}, \boldsymbol{\theta}_{1}, \mathcal{P}_{1}\right), \ldots,\left(\boldsymbol{\alpha}_{c}, \boldsymbol{\theta}_{c}, \mathcal{P}_{c}\right)\right\}=\operatorname{Partition}(\boldsymbol{\alpha}, \boldsymbol{\theta}, \mathcal{P})$,

5.2) For each partition $l$ from 1 to $c$

5.2.1) $\boldsymbol{y}_{l}=\boldsymbol{y}-\sum_{\substack{j=1 \\ j \neq l}}^{c} \sum_{i=1}^{K_{j}} \alpha_{j, i} \psi\left(\theta_{j, i} ; \boldsymbol{t}\right)$

5.2.2) $\left[\boldsymbol{\alpha}_{l}, \boldsymbol{\theta}_{l}\right]=\mathcal{S}\left(\boldsymbol{y}_{l}, K_{l}, \mathcal{P}_{l}\right)$

5.3) $\boldsymbol{\alpha}=\bigcup_{j, i} \alpha_{j, i}, \boldsymbol{\theta}=\bigcup_{j, i} \theta_{j, i}$, combine,

6) $\hat{\boldsymbol{\alpha}}=\boldsymbol{\alpha}$ and $\hat{\boldsymbol{\theta}}=\boldsymbol{\theta}$, finalize the solution.

angular frequency, respectively; $\boldsymbol{n}$ is a zero mean circularly symmetric i.i.d. complex Gaussian noise with variance $\sigma^{2}$, and $t$ is the vector of sampling times.

In the frequency estimation problem, 1 -sparse case is similar to the single tone frequency estimation problem. In the regular sampling, this is a well studied problem [11]. However, to best of our knowledge there is no reported study on the CRLB of the single tone frequency estimation under random sampling.

In (20), important difference from the regular sampling is that time sampling is also random. In this case, CRLB for the single tone frequency estimation under the random sampling, whose derivation is omitted due to limited space, is:

$$
\operatorname{var}(\widehat{\omega}) \geqslant \frac{(\sigma / \alpha)^{2}}{M} \frac{1}{\operatorname{var}\left(t_{i}\right)},
$$

where $\operatorname{var}\left(t_{i}\right)$ is the variance of the random time samples. When sampling times are generated from an i.i.d. uniform distribution in the $[0, T]$ range, CRLB of the frequency estimation becomes:

$$
\operatorname{var}(\widehat{\omega}) \geqslant J_{u}=\frac{(\sigma / \alpha)^{2}}{M} \frac{12}{T^{2}},
$$

which is asymptotically equivalent to the CRLB in the regular sampling case [11].

If we use an unbiased estimator of the parameters, squareroot of the CRLB can be thought as the finest partition size that the estimator can achieve under the assumed noise statistics. Even though (14) allows arbitrarily dense discretization in the 1-sparse case, intervals smaller than square-root of the CRLB will not provide any further improvement in the estimation performance. Therefore, treatment to this important case is to re-define (13) for $K=1$ as a fraction of (22).

\section{B. Sparse Solver}

The proposed framework can be used with any sparse solver of choice. However, algorithms with low complexity are preferable since the proposed framework recursively initiates several instances of the same problem. More importantly, selected sparse solver is expected to be a minimum variance unbiased estimator (MVUE) of the parameters for a sparsity level so that the proposed framework achieves the CramérRao lower bound in the estimation variance. OMP with dense discretization has this property at $K=1$ [11].

\section{Satisfactory Solution}

One straightforward way to terminate the iterations is to observe the residual error. Using a predefined threshold $\epsilon_{1}$, the provisional solution, $(\boldsymbol{\alpha}, \boldsymbol{\theta})$, can be qualified as satisfactory if $\left\|\boldsymbol{y}-\boldsymbol{y}^{*}\right\|_{2} \leqslant \epsilon_{1}$, where $\boldsymbol{y}^{*}$ is the reconstructed observation. Another approach is to monitor the residual norm and terminate the iterations when rate of decrease in the residual is below a certain threshold $\epsilon_{2},\left\|\boldsymbol{y}_{q-1}^{*}\right\|_{2} /\left\|\boldsymbol{y}_{q}^{*}\right\|_{2} \leqslant \epsilon_{2}$, where $q$ is the index of iterations. Also, total number of iterations can be bounded. For a robust behavior, some of the discussed metrics can be used in conjunction.

\section{Partitioning}

Splitting the problem into self-similar sub-problems requires a partitioning operation on the provisional solution set. In the proposed framework any clustering algorithm can be implemented to partition the original problem into subproblems. However, to reduce the required computational load, we propose to use fixed $c=2$, and split the main problem into two distinct parts as "the most dominant" and "the rest". Thus, in each recursive call, problem will be split into two sub-problems with sparsity 1 and $K-1$.

\section{E. Parallelization}

Due to sequential solutions in sub-problems, current form of the algorithm is not suitable for parallelization. However, it is possible to solve each sub-problem independently from each other. Independent solutions can run on different processors resulting in savings on the computational time. Although not reported here, preliminary investigations indicate that the obtained results are in close proximity of the CRLB.

\section{Simulations}

In this section, performance of the proposed framework is investigated in sparse spectral estimation. The observation vector with $K$-sparse components is constructed as:

$$
\boldsymbol{y}=\sum_{i=1}^{K} \alpha_{i} e^{j \phi_{i}} e^{j 2 \pi f_{i} \boldsymbol{t}}+\boldsymbol{n}
$$

where $t \in \Re^{M}$ is constructed by selecting time samples uniformly from $[0, T]$ range with $T=1 \mathrm{~s}$. $\phi_{i}$ 's are selected uniformly in $[0,2 \pi]$ range and $\alpha_{i}=1$ for $1 \leqslant i \leqslant K$. The frequency of the components, $f_{i}$, are selected uniformly random in $[100,300] \mathrm{Hz}$ range; $\boldsymbol{n} \in \mathbb{C}^{M}$ is i.i.d. complex Gaussian noise with zero mean and standard deviation $\sigma$. In the following parts, $\alpha / \sigma$ will be considered as the measure of Signal-to-Noise Ratio (SNR).

OMP and CoSaMP [12] are compared to their recursive counterparts in the proposed framework. Even though OMP with a fine grid has been reported with a limited performance gain [13], the results of OMP with a dense grid is also 
provided for the comparison purposes. Together with the standard deviations of the error in the estimated frequencies, the CRLB given in (22) is presented in Fig. 2.

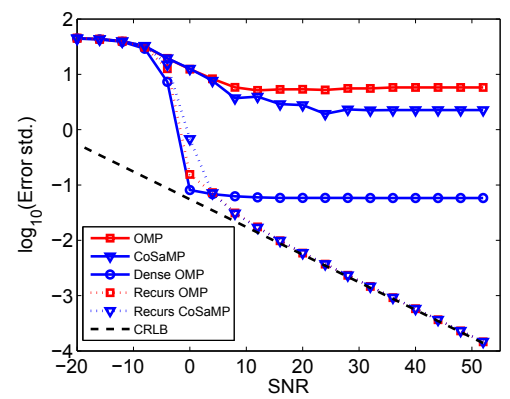

(a)

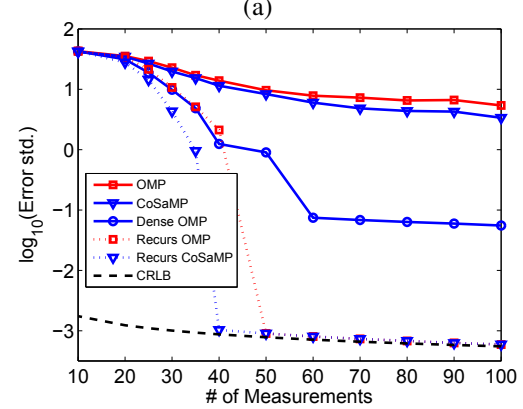

(b)

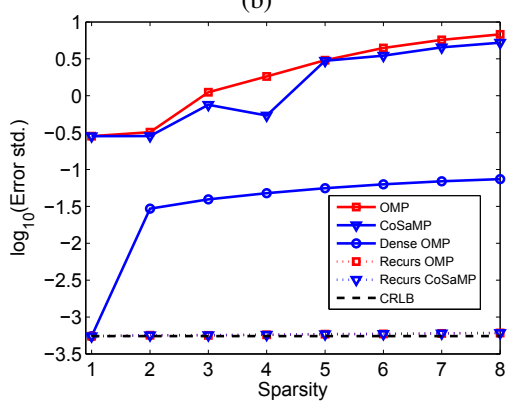

(c)

Fig. 2. Standard deviation of the error in the solution of the frequency (a) w.r.t. $S N R$ at $K=5, M=100$, (b) w.r.t. number of measurements at $K=5$, $\mathrm{SNR}=40 \mathrm{~dB}$, (c) w.r.t. sparsity at $\mathrm{M}=100, \mathrm{SNR}=40 \mathrm{~dB}$

In Fig. 2(a), the proposed framework is tested against various level of SNR. Regular solvers and their recursive counterparts have a transition around $0 \mathrm{~dB}$. For $\mathrm{SNR}<0 \mathrm{~dB}$, regular and their recursive counterparts behave similarly with a significant deviation from the CRLB. When SNR is higher than $0 \mathrm{~dB}$, there is a little improvement in the regular solvers. Due to the off-grid problem, solvers do not provide significant improvements even at high SNR. For OMP, a denser grid brings a reduction in the error variance. Yet, the improved estimation performance is still far from the CRLB. Error variance of the solvers in the proposed framework scales down with the noise variance achieving the CRLB for SNR's greater than $10 \mathrm{~dB}$. In Fig.2(b), the same behavior is observed for the varying number of measurements. The Nyquist rate sampling would require 400 samples, whereas regular solvers and their recursive counterparts have similar break points around $10-12 \%$ of the Nyquist rate samples, i.e. $M \approx 45$.
In Fig.2(c) the proposed framework is tested against various level of sparsity. In the regular solvers, error variance increases with the sparsity level. When $K=1$, OMP with a denser grid coincides with the base case of the recursion and achieves the CRLB. For sparsity $K>1$, denser grid again provides a limited increase in the estimation performance for OMP. The recursive solvers, on the other hand, provide significantly better estimates achieving CRLB compared to their nonrecursive counterparts.

\section{Conclusions}

In this paper, a novel recursive framework is presented. The proposed framework partitions the original problem into sparser sub-problems and discretizes the given continuous parameter space adaptively depending on the sparsity of the problem in order to guarantee the reconstruction with the specified sparse solver. The performance of the proposed framework is illustrated in a sparse spectral estimation problem. Results indicate that, in comparison with the direct use of a solver, its recursive implementation in the proposed framework can result significantly lower error variances achieving the CRLB. Due to its modular structure, the proposed framework is highly flexible and can conduct its iterations using any solver of choice. Also, its parallelizable structure can be exploited for improved performance/complexity gains in multi-processors systems.

\section{REFERENCES}

[1] D. Donoho, "Compressed sensing," IEEE Trans. Information Theory, vol. 52, no. 4, pp. 1289-1306, 2006.

[2] E. Candes, J. Romberg, and T. Tao, "Robust uncertanity principles: Exact signal reconstruction from highly incomplete frequency information," IEEE Trans. Information Theory, vol. 52, pp. 489-509, 2006.

[3] G. Tang, B. N. Bhaskar, P. Shah, and B. Recht, "Compressed sensing off the grid," Arxiv, vol. abs/1207.6053, 2012.

[4] M. Herman and T. Strohmer, "General deviants: An analysis of perturbations in compressed sensing," IEEE Journal of Selected Topics in Signal Processing, vol. 4, no. 2, pp. 342 - 349, 2010.

[5] H. Zhu, G. Leus, and G. Giannakis, "Sparsity-cognizant total leastsquares for perturbed compressive sampling," IEEE Trans. on Signal Processing, vol. 59, no. 5, pp. 2002 - 2016, 2011.

[6] O. Teke, A. C. Gurbuz, and O. Arikan, "A robust compressive sensing based technique for reconstruction of sparse radar scenes," Digital Signal Processing, vol. 27, no. 0, pp. 23 - 32, 2014.

[7] R. Baraniuk, M. Davenport, R. Devore, and M. Wakin, "A simple proof of the restricted isometry property for random matrices," Constr. Approx, vol. 2008, 2007.

[8] J. Tropp, "Greed is good: Algorithmic results for sparse approximation," IEEE Trans. Information Theory, vol. 50, no. 10, pp. 2231-2242, Oct. 2004.

[9] A. Dempster, N. Laird, and D. Rubin, "Maximum likelihood from incomplete data via the em algorithm," Journal of the Royal Statistical Societys Series B (Methodological), vol. 39, no. 1, pp. 1-38, 1977.

[10] A. Gurbuz, M. Pilanci, and O. Arikan, "Expectation maximization based matching pursuit," in Acoustics, Speech and Signal Processing (ICASSP), 2012 IEEE International Conference on, March 2012, pp. 3313-3316.

[11] D. Rife and R. Boorstyn, "Single tone parameter estimation from discrete-time observations," Information Theory, IEEE Transactions on, vol. 20, no. 5, pp. 591-598, Sep 1974.

[12] D. Needell and J. Tropp, "Cosamp: Iterative signal recovery from incomplete and inaccurate samples," Applied and Computational Harmonic Analysis, vol. 26, no. 3, pp. $301-321,2009$.

[13] O. Teke, A. Gurbuz, and O. Arikan, "Perturbed orthogonal matching pursuit," Signal Processing, IEEE Transactions on, vol. 61, no. 24, pp. 6220-6231, 2013. 